\title{
Rhamnosidase activity of selected probiotics and their ability to hydrolyse flavonoid rhamnoglucosides
}

\author{
Monika Mueller $^{1} \cdot$ Barbara Zartl $^{1} \cdot$ Agnes Schleritzko $^{1} \cdot$ Margit Stenzl $^{1}$ • \\ Helmut Viernstein ${ }^{1} \cdot$ Frank M. Unger ${ }^{1}$
}

Received: 12 October 2017 / Accepted: 24 October 2017 / Published online: 10 November 2017

(c) The Author(s) 2017. This article is an open access publication

\begin{abstract}
Bioavailability of flavonoids is low, especially when occurring as rhamnoglucosides. Thus, the hydrolysis of rutin, hesperidin, naringin and a mixture of narcissin and rutin (from Cyrtosperma johnstonii) by 14 selected probiotics was tested. All strains showed rhamnosidase activity as shown using 4-nitrophenyl $\alpha$-L-rhamnopyranoside as a substrate. Hesperidin was hydrolysed by $8-27 \%$ after 4 and up to $80 \%$ after 10 days and narcissin to $14-56 \%$ after 4 and $25-97 \%$ after 10 days. Rutin was hardly hydrolysed with a conversion rate ranging from 0 to $5 \%$ after 10 days. In the presence of narcissin, the hydrolysis of rutin was increased indicating that narcissin acts as an inducer. The rhamnosidase activity as well as the ability to hydrolyse flavonoid rhamnoglucosides was highly strain specific. Naringin was not hydrolysed by rhamnosidase from probiotics, not even by the purified recombinant enzyme, only by fungal rhamnosidase. In conclusion, rhamnosidases from the tested probiotics are substrate specific cleaving hesperidin, narcissin and to a small extent rutin, but not naringin.
\end{abstract}

Keywords Rhamnosidase $\cdot$ Hesperidin $\cdot$ Naringin · Rutin $\cdot$ Narcissin $\cdot$ Probiotics

Electronic supplementary material The online version of this article (https://doi.org/10.1007/s00449-017-1860-5) contains supplementary material, which is available to authorized users.

Monika Mueller

monika.mueller@univie.ac.at

Department of Pharmaceutical Technology and Biopharmaceutics, University of Vienna, Althanstrasse 14, 1090 Vienna, Austria

\section{Introduction}

As secondary plant metabolites with important contents in human diets $[1,2]$, flavonoids occur in a wide variety of compounds comprising six subclasses [3, 4]. Flavonols, the most common flavonoids in foods include quercetin, its glycoside rutin (quercetin-3-rutinoside), kaempferol, isorhamnetin and its glycoside narcissin (isorhamnetin-3-rutinoside) $[5,6]$. Main sources of these compounds are onions and broccoli, but also red wine and tea. Flavanones with their important representatives naringenin (grapefruits) and hesperetin (oranges), and their glycosides, are also found in other human foods such as tomatoes and aromatic plants [4]. The most familiar flavones in diet are apigenin and luteolin which occur in red pepper [5] and celery [5, 7]; isoflavones are present in soybean-derived products or red clover and show commonalities with estrogens [8] and flavanols occur in apricots, red wine, green tea and chocolate [4].

Interest in flavonoids has been growing because of their biological properties such as anti-inflammatory, antioxidant, antimutagenic, antiproliferative and antiatherogenic effects as shown for quercetin, isorhamnetin, naringenin, hesperetin and its glycosides rutin, narcissin, naringin (naringenin-7-neohesperidoside) and hesperidin (hesperetin7-rutinoside) [9-12]. Thus, those compounds play a role in amelioration of various diseases or disorders with high prevalence globally including neurodegenerative diseases, osteoporosis, cancer or cardiovascular diseases including atherosclerosis, thrombosis and hypertension.

Most flavonoids are found in glycosylated form, often glycosylated by rutinose (6- $O$ - $\alpha$-L-rhamnopyranosyl-Dglucose), as rutinosides (e.g. rutin, hesperidin, narcissin) or by neohesperidose (2- $O$ - $\alpha$-L-rhamnopyranosyl-D-glucose) as neohesperidosides (e.g. naringin). Glycosylation by rutinose or neohesperidose hinders the absorption of the 
flavonoids in the small intestine [6]. Due to a lack of human intestinal $\alpha$-L-rhamnosidase, a prior enzymatic hydrolysis increases bioavailability and bioactivity of flavonoid glycosides [13-15]. $\alpha$-L-Rhamnosidase [EC 3.2.1.40] cleaves terminal $\alpha$-L-rhamnose from several natural products [16] and is applied for de-bittering of fruit juices [17] or enhancing wine aroma [18]. Beside its biotechnological applications, there is an upcoming interest in $\alpha$-L-rhamnosidase for enhancing the bioavailability of natural glycosides such as flavonoids rhamnoglucosides by hydrolysing the L-rhamnose [19]. Due to their wide availability, high selectivity, low cost and fast reaction behaviour, the use of enzymes to modify the structure and to improve the physicochemical and biological properties of flavonoids has become an interesting alternative [15]. Enzymes from probiotics have the advantage of safety and possible combined health effects of polyphenols and probiotics [20].

Here, we tested the rhamnosidase activity of 14 selected probiotic strains and their ability to hydrolyse naringin, hesperidin, rutin and the main constituents (rutin and narcissin) of an ethanolic extract of Cyrtosperma johnstonii which exerts antioxidant activity and cytotoxicity towards cancer cells as previously shown [21].

\section{Materials and methods}

\section{Chemicals, plant extract and bacterial strains}

Acetonitrile was purchased from Promochem (Wesel, Germany), recombinant $\alpha$-L-rhamnosidase from prokaryotic source $(190 \mathrm{U} / \mathrm{mg}$ ) was obtained in purified form from Megazyme (Wicklow, Ireland), $\beta$-glycosidase/ $\alpha$-Lrhamnosidase mixture [Cellulase from $(A)$ niger, $0.45 \mathrm{U} /$ $\mathrm{mg}$ ] from Sigma-Aldrich (St. Louis, MO, USA), flavonoid glycosides, aglycones and all other chemicals from Sigma-Aldrich [or Merck (Darmstadt, Germany)]. Dried powder of $C$. johnstonii rhizomes (voucher specimen No 010112, gift of Chiang Mai University, Thailand) was extracted with ethanol for $24 \mathrm{~h}$, filtrated and evaporated to obtain a dried crude extract. The probiotic strains used in this study included Lactobacillus (L.) paracasei ssp. paracasei CRL 431 (ATCC 55544), L. paracasei ssp. paracasei (DN114001), L. paracasei ssp. paracasei DSM 20312 (Shirota), L. rhamnosus GG (ATCC 53103), L. reuteri (ATCC 55730), L. acidophilus LA-5 (DSM 13241), Bifidobacterium (B.) animalis ssp. lactis BB12 (DSM 15954), (B) longum ssp. infantis, L. plantarum (ATCC 15697), L. brevis (ATCC 367), L. delbrueckii ssp. bulgaricus (DSM 20081), Lactococcus (Lc.) lactis ssp. lactis (SR 3.54; NCIMB 30117), L. fermentum, Streptococcus $(S$.) salivarius ssp. thermophilus (ATCC 19258). Man-Rogosa-Sharpe (MRS) liquid medium was prepared as described previously [22].
Hydrolysis of the flavonoid rhamnoglucosides using rhamnosidase

As a positive control, glycosides $\left(10^{-4} \mathrm{M}\right)$ were incubated with a recombinant $\alpha$-L-rhamnosidase $(1 \mathrm{U} / \mathrm{mL})$ or an enzyme mixture from $A$. niger $(5 \mathrm{U} / \mathrm{mL} \beta$-glucosidase with rhamnosidase side activity) in $100 \mathrm{mM}$ sodium phosphate buffer $\mathrm{pH}=6.5$ at $37{ }^{\circ} \mathrm{C}$ for up to $3 \mathrm{~h}$. The negative control was incubated without enzyme; 4-nitrophenyl $\alpha$-Lrhamnopyranoside (NPRP) was used as reference substance. Samples were drawn at certain time points, diluted with methanol (1:1) to precipitate the enzymes and clarified using centrifugation at $13,000 \mathrm{rpm}$ and $4{ }^{\circ} \mathrm{C}$ for $15 \mathrm{~min}$. Supernatants were analysed using high performance liquid chromatography (HPLC). All experiments were performed in independent triplicates.

\section{Analysis of the hydrolysis of flavonol rutinosides by probiotics}

Probiotics were cultivated overnight in MRS medium with glucose under anaerobic conditions at $37{ }^{\circ} \mathrm{C}$, diluted with MRS medium without carbohydrates to an $\mathrm{OD}_{600}$ of 0.2 and further incubated for $48 \mathrm{~h}$. Subsequently, the bacterial suspension was diluted to an $\mathrm{OD}_{600}$ of 0.2 in MRS medium without carbohydrate source, supplemented with $10^{-4}$ M NPRP, nitrophenyl $\beta$-D-glucopyranoside (NPGP), rutin, naringin, hesperidin or with extract of $C$. johnstonii (main compounds $\sim 10^{-4} \mathrm{M}$ ) and incubated at $37^{\circ} \mathrm{C}$ for up to 10 days under anaerobic conditions. Samples of NPRP were drawn after $0,1,2,3,4,7$ days and samples of the rutinosides after $0,4,7$ and 10 days. The negative control was incubated without bacteria for 10 days. Samples were diluted with methanol (1:1) and clarified using centrifugation at $13,000 \mathrm{rpm}$ and $4{ }^{\circ} \mathrm{C}$ for $15 \mathrm{~min}$. Supernatants were analysed using HPLC. All experiments were performed in triplicates.

\section{Analysis of flavonols and their glycosides by HPLC}

The extent of hydrolysis was determined using HPLC with ultraviolet detection (HPLC-UV) on an UltiMate3000 HPLC system (Thermo Fisher Scientific, Waltham, MA, USA) connected to a security guard cartridge followed by a Kinetex C-18 column ( $5 \mu \mathrm{m} \mathrm{C18}, 4.6 \times 150 \mathrm{~mm}$, Phenomenex, Torrance, CA, USA). The mobile phase consisted of solvent A (water-acetonitrile 95:5, 0.1\% trifluoroacetic acid, TFA) and solvent B (acetonitrile, 0.1\% TFA). A linear gradient from 0 to $100 \%$ B was applied with a flow rate of $0.5 \mathrm{~mL} /$ min: from 0 to $2.5 \mathrm{~min} B$ was increased to $15 \%$; from 2.5 to $7.5 \mathrm{~min}$ B was increased to $20 \%$, until $13.5 \mathrm{~min}$ B was increased to $25 \%$; until 14 min B was increased to $30 \%$; until $15 \mathrm{~min}$ to $35 \%$; until $17 \mathrm{~min}$ to $50 \%$; until $22 \mathrm{~min}$ to 
$100 \%$; after 2 min at $100 \%$, the column was equilibrated with $100 \%$ A. The elution profile was recorded using a photodiode array detector PDA-100 set at the respective absorption maxima of the compounds (305 $\mathrm{nm}$ for NPRP, NPGP and nitrophenol; $280 \mathrm{~nm}$ for naringenin, hesperetin and their glycosides, and $350 \mathrm{~nm}$ for quercetin, isorhamnetin and their glycosides). The respective retention times were $11.8 \mathrm{~min}$ for NPRP, 7.7 min for NPGP and 12.8 for nitrophenol; 12.9 for rutin, 13.6 for isoquercitrin and 22.1 for quercetin; 14.6 for naringin and 23.8 for naringenin; 15.8 for hesperidin and 24.3 for hesperetin; 15.7 for isorhamnetinrutinosid, 16.6 for isorhamnetin-3-O-glucoside and 24.6 for isorhamnetin. Flavonoids and their glycosides were quantified using Chromeleon software. The results are shown as mean (from a triplicate) \pm standard deviation.

\section{Analysis of rhamnosidase by SDS-PAGE}

The rhamnosidase expression was detected in the cell lysates of $L$. acidophilus as an example and the molecular weight was compared to the recombinant rhamnosidase and the enzyme mixture from $A$. niger using sodium dodecyl sulphate polyacrylamide gel electrophoresis (SDS-PAGE). Bacterial culture samples were taken at different time points and centrifuged at $12,500 \mathrm{rpm}$ and RT for $10 \mathrm{~min}$. The pellets were re-suspended in diluted cell lysis reagent (CelLytic ${ }^{\mathrm{TM}} \mathrm{B}$ lysis reagent, Sigma Aldrich) and incubated $10 \mathrm{~min}$ at RT under mixing at $350 \mathrm{rpm}$. Samples were centrifuged (12,500 rpm, $10 \mathrm{~min}, \mathrm{RT})$ and the supernatants were diluted with Laemmli sample buffer (BIO-RAD). Samples were incubated at $99{ }^{\circ} \mathrm{C}$ for $10 \mathrm{~min}$, mixed at $350 \mathrm{rpm}$ and afterward loaded to the gel (12\% Mini-PROTEAN ${ }^{\circledR}$ TGX $^{\mathrm{TM}}$ precast protein gels, 15 -well, $15 \mu \mathrm{L}, \mathrm{BIO}-\mathrm{RAD})$ and run with electrophoreses buffer (10× Tris/Glycine/SDS, BIO-RAD) at $130 \mathrm{~V}$. As protein marker peqGOLD protein marker IV (10-170 kDa, VWR) was used. Proteins were stained with Coomassie brilliant blue R-250.

\section{Results}

\section{Hydrolysis of flavonoid rhamnoglucosides using purified rhamnosidases}

The recombinant rhamnosidase (used as positive control) was highly efficient for the hydrolysis of the flavonoid glycosides and NPRP (Table 1). 50\% of rutin were hydrolysed into isoquercitrin after $20 \mathrm{~min}$ and $100 \%$ after $130 \mathrm{~min}$ at an incubation temperature of $37{ }^{\circ} \mathrm{C}$. Naringin was not hydrolysed. Hesperidin showed a lower hydrolysis rate than rutin with $50 \%$ of conversion to hesperetin-7- $O$-glucoside after 90 min, whereas a $100 \%$ hydrolysis was not reached. No hydrolysis was observed in the negative controls without enzymes for up to $72 \mathrm{~h}$. The hydrolysis of all tested flavonoid glycosides was higher at $50^{\circ} \mathrm{C}$.

Rhamnosidase from $A$. niger is capable of hydrolysing also naringin to naringenin-7-O-glucoside. Using this enzyme, results in the most efficient cleavage of hesperidin followed by naringin and rutin (Table 1).

\section{Rhamnosidase and $\beta$-glucosidase activity of probiotics}

All probiotic strains exerted a significant rhamnosidase activity as indicated using NPRP as a substrate leading to more than $40 \%$ hydrolysis for 8 out of 14 strains after 1 day under anaerobic conditions (Table 2; Fig. 1A). In general, the hydrolysis rate of NPRP ranged between $20 \%$ (L. reuteri) and $68 \%$ [Lactococcus (Lc.) lactis] after 1 day of incubation and reached a total of $>86 \%$ hydrolysis after 4 days with exception of $L$. reuteri. The highest rhamnosidase activity after 1 day was exerted by Lc. lactis followed by L. fermentum and L. brevis, followed by L. rhamnosus GG, B. longum ssp. infantis and L. paracasei ssp. paracasei DSM 20312. The lowest rhamnosidase activity after 1 day was exerted by $L$. reuteri, followed by $L$. delbrueckii ssp. bulgaricus, and $S$. salivarius ssp. thermophilus.

The $\beta$-glucosidase activity is higher than the rhamnosidase activity. In fact, NPGP is converted into the aglycone to an extent of 94-100\% already after $4 \mathrm{~h}$ (except L. rhamnosus GG) and totally converted after 1 day by all 14 included strains (Table 2; supplements).
Table 1 Hydrolysis of rhamnose from NPRP, rutin, naringin and hesperidin by rhamnosidases from different sources: time after which 50 or $100 \%$ of the glycosides were cleaved, $n=3$

\begin{tabular}{|c|c|c|c|c|c|c|c|c|}
\hline \multirow[t]{3}{*}{ Hydrolysis } & \multicolumn{4}{|c|}{ Recombinant rhamnosidase } & \multicolumn{4}{|c|}{ Rhamnosidase from A. niger } \\
\hline & \multicolumn{2}{|l|}{$37^{\circ} \mathrm{C}$} & \multicolumn{2}{|l|}{$50{ }^{\circ} \mathrm{C}$} & \multicolumn{2}{|l|}{$37^{\circ} \mathrm{C}$} & \multicolumn{2}{|l|}{$50{ }^{\circ} \mathrm{C}$} \\
\hline & $50 \%$ & $100 \%$ & $50 \%$ & $100 \%$ & $50 \%$ & $100 \%$ & $50 \%$ & $100 \%$ \\
\hline NPRP & $<5 \min$ & $\sim 20 \mathrm{~min}$ & $<5 \min$ & $\sim 20 \mathrm{~min}$ & $\sim 10 \mathrm{~h}$ & $\sim 48 \mathrm{~h}$ & $\sim 6 \mathrm{~h}$ & $\sim 48 \mathrm{~h}$ \\
\hline Rutin & $20 \min$ & $130 \mathrm{~min}$ & $15 \mathrm{~min}$ & $170 \min$ & $\sim 35 \mathrm{~h}$ & - & $\sim 22 \mathrm{~h}$ & - \\
\hline Naringin & - & - & - & - & $<6 \mathrm{~h}$ & $<6 \mathrm{~h}$ & $<6 \mathrm{~h}$ & $<6 \mathrm{~h}$ \\
\hline Hesperidin & $90 \mathrm{~min}$ & - & $70 \mathrm{~min}$ & - & $\sim 10 \mathrm{~h}$ & - & $<6 \mathrm{~h}$ & - \\
\hline
\end{tabular}


Table 2 Hydrolysis of rhamnose (bioconversion) from NPRP, rutin, naringin and hesperidin or hydrolysis of glucose from NPGP at certain time points. The table lists the bioconversion in \%

\begin{tabular}{|c|c|c|c|c|c|c|c|c|}
\hline \multirow[t]{3}{*}{ Incubation } & \multicolumn{8}{|c|}{ Bioconversion (\%) } \\
\hline & \multicolumn{2}{|l|}{ NPRP } & \multirow{2}{*}{$\begin{array}{l}\text { NPGP } \\
4 \mathrm{~h}\end{array}$} & \multirow{2}{*}{$\begin{array}{l}\text { Rutin } \\
10 \text { days }\end{array}$} & \multirow{2}{*}{$\begin{array}{l}\text { Naringin } \\
10 \text { days }\end{array}$} & \multicolumn{3}{|c|}{ Hesperidin } \\
\hline & 1 day & 4 days & & & & 4 days & 7 days & 10 days \\
\hline B. animalis ssp. lactis $\mathrm{BB} 12$ & $48 \pm 0$ & $88 \pm 7$ & 96 & 0 & 0 & $23 \pm 3$ & $52 \pm 4$ & $69 \pm 9$ \\
\hline B. longum ssp. infantis & $50 \pm 0$ & $95 \pm 3$ & 100 & 0 & 0 & $17 \pm 3$ & $52 \pm 11$ & $54 \pm 8$ \\
\hline L. acidophilus LA-5 & $33 \pm 4$ & $87 \pm 6$ & 100 & $3 \pm 0$ & 0 & $27 \pm 1$ & $58 \pm 3$ & $84 \pm 7$ \\
\hline L. paracasei ssp. paracasei DN114001 & $43 \pm 0$ & $88 \pm 5$ & 100 & 0 & 0 & $8 \pm 3$ & $15 \pm 4$ & $25 \pm 1$ \\
\hline L. paracasei ssp. paracasei DSM 20312 & $49 \pm 0$ & $92 \pm 8$ & 100 & 0 & 0 & $20 \pm 3$ & $36 \pm 8$ & $56 \pm 4$ \\
\hline L. paracasei ssp. paracasei CRL 431 & $33 \pm 9$ & $90 \pm 6$ & 97 & 0 & 0 & $13 \pm 2$ & $34 \pm 4$ & $55 \pm 11$ \\
\hline L. reuteri & $20 \pm 6$ & $74 \pm 15$ & 100 & 0 & 0 & $3 \pm 1$ & $12 \pm 7$ & $17 \pm 7$ \\
\hline L. rhamnosus GG & $52 \pm 8$ & $97 \pm 3$ & 28 & 0 & 0 & $6 \pm 1$ & $8 \pm 5$ & $18 \pm 5$ \\
\hline L. plantarum & $32 \pm 4$ & $98 \pm 1$ & 100 & $5 \pm 0$ & 0 & $11 \pm 6$ & $46 \pm 6$ & $70 \pm 8$ \\
\hline L. brevis & $63 \pm 5$ & $100 \pm 0$ & 100 & 0 & 0 & $25 \pm 3$ & $53 \pm 4$ & $80 \pm 5$ \\
\hline L. delbrueckii ssp. bulgaricus & $25 \pm 9$ & $98 \pm 0$ & 100 & $2 \pm 0$ & 0 & $21 \pm 9$ & $34 \pm 8$ & $48 \pm 8$ \\
\hline Lc. lactis ssp. lactis & $68 \pm 0$ & $100 \pm 0$ & 95 & 0 & 0 & $15 \pm 2$ & $39 \pm 5$ & $57 \pm 1$ \\
\hline L. fermentum & $63 \pm 2$ & $100 \pm 0$ & 94 & 0 & 0 & $21 \pm 1$ & $43 \pm 1$ & $66 \pm 5$ \\
\hline S. salivarius ssp. thermophilus & $26 \pm 3$ & $86 \pm 6$ & 100 & $4 \pm 0$ & 0 & $18 \pm 5$ & $43 \pm 6$ & $55 \pm 5$ \\
\hline
\end{tabular}

This is defined of the percentages of rhamnose cleaved from the glycosides, $n=3$

\section{Hydrolysis of rutin, hesperidin and naringin by probiotics}

The rate of hydrolysis of rhamnose from flavonoid rhamnoglucosides was highly dependent on the aglycon (Table 2). After cleavage of the rhamnose, the glucose is cleaved rapidly.

Rutin was hardly hydrolysed by probiotic rhamnosidases with a conversion (to isoquercitrin, following by hydrolysis to quercetin) ranging from 0 to $2 \%$ after 4 days, 0-3\% after 7 days and 0-5\% after 10 days by four strains, namely $L$. plantarum, L. delbrueckii ssp. bulgaricus, $S$. salivarius ssp. thermophilus and L. acidophilus.

The conversion rate of hesperidin was significantly higher ranging from $8 \%$ (L. paracasei DN114001) to 27\% (L. acidophilus) after 4 days, 9\% (L. rhamnosus GG) up to $54 \%$ (L. brevis) after 7 days and 25 up to $80 \%$ (L. paracasei DN114001) after 10 days (L. brevis) (Table 2). Hesperidin was hydrolyzed most efficiently by $L$. acidophilus, followed by L. brevis and B. animalis ssp. lactis. The hydrolysis of hesperidin was least efficient by $L$. reuteri and $L$. rhamnosus GG. Since the hydrolysis of the glucose after the hydrolysis of the rhamnose is much faster, only the aglycone hesperetin but not the hesperetin-7-O-glucoside can be found (Fig. 1B, supplements).

Naringin was not hydrolysed by any of the used probiotic bacteria.

\section{Cleavage of rhamnoglucosides from $C$. johnstonii extract by probiotics}

The extract of $C$. johnstonii contains rutin and narcissin as main components. Narcissin is converted significantly faster as rutin or hesperidin. In fact, narcissin is converted by $14 \%$ (L. reuteri) to $56 \%$ (L. acidophilus) after 4 days, by $15 \%$ (L. reuteri) to $87 \%$ (L. fermentum) after 7 days and to more than $80 \%$ by ten strains after 10 days (Table 3 ). The glucoside (isorhamnetin-3-glucoside) and aglycone (isorhamnetin) can be detected in parallel. As expected, the relative amount of aglycone to glucoside increases with the duration of incubation (Fig. 1C, supplements).

Interestingly, in the presence of narcissin, the rhamnosidase activity is induced, so that the conversion of rutin is enabled or enhanced (Table 3; Fig. 1D, supplements). In fact, rutin is converted by up to $15 \%$ after 4 days (B. infantis), up to $51 \%$ after 7 days (L. fermentum) and up to $78 \%$ after 10 days (L. fermentum). Rhamnosidase activity was induced most efficiently in $L$. fermentum, B. longum ssp. infantis and Lc. lactis. However, rhamnosidase was hardly induced in $L$. reuteri. The hydrolysis products isoquercitrin and quercetin can be found in parallel with increasing amounts of quercetin with longer incubation duration. 
A

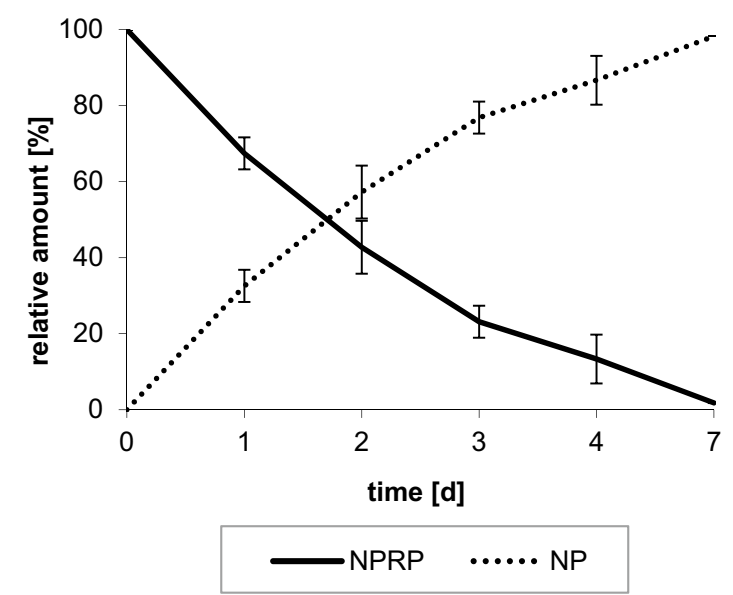

C

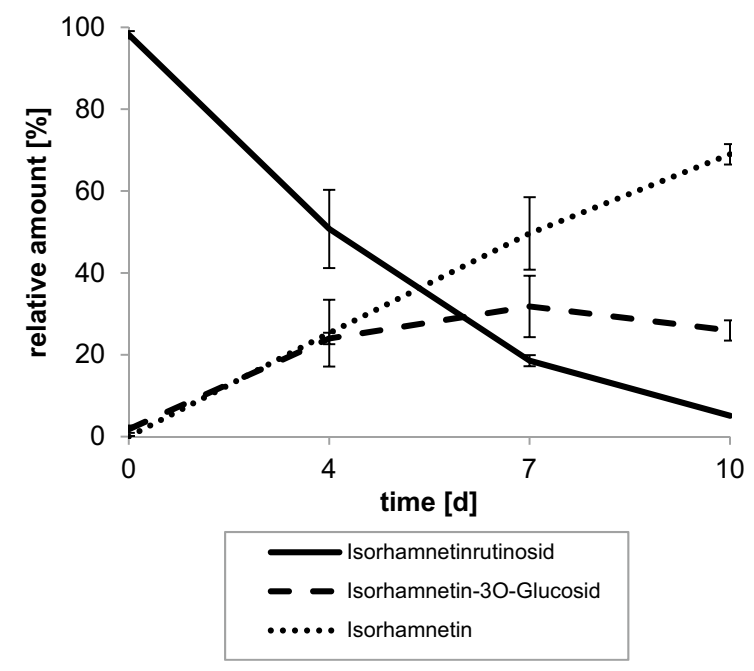

B

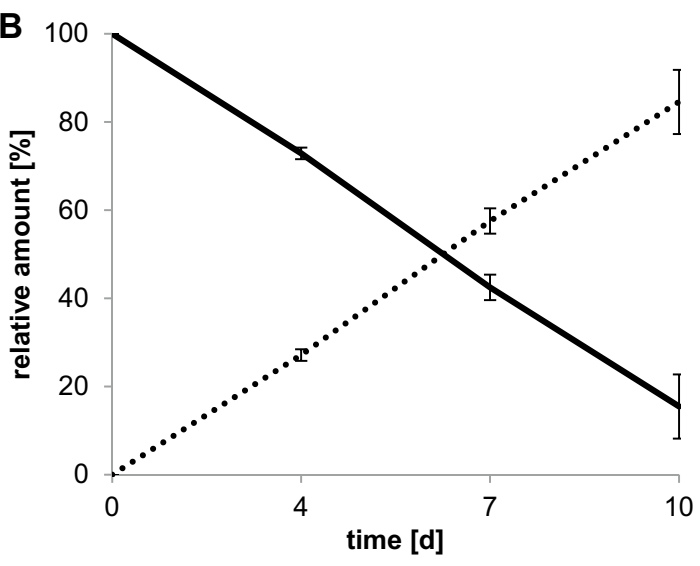

- Hesperidin ...... Hesperetin

D

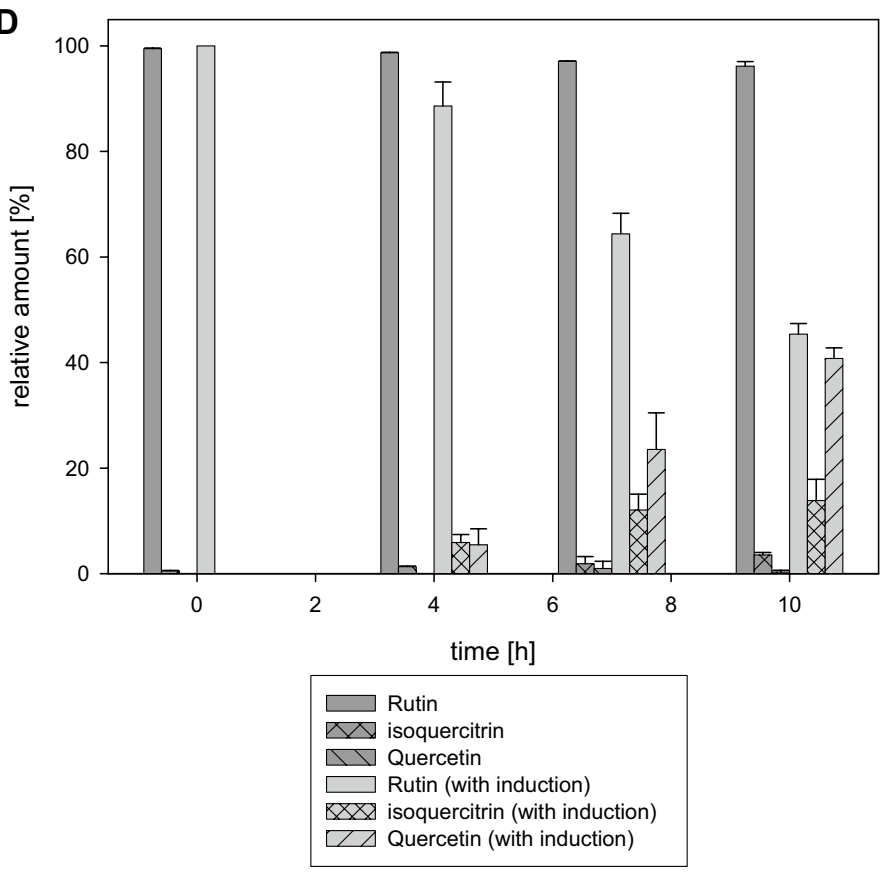

Fig. 1 Rhamnosidase activity of L. acidophilus (as example). Deglycosylation of A NPRP, B hesperidin, C narcissin and D rutin without and with induction by narcissin in an extract of $C$. johnstonii

\section{Expression of rhamnosidase in probiotics}

The expression of rhamnosidase is induced by the substrates as shown with NPRP in L. acidophilus (Fig. 2). It could be shown that the amount of rhamnosidase increases with the incubation time. The probiotic rhamnosidase was found to have a molecular weight of $80-90 \mathrm{kDa}$ which is similar to the recombinant rhamnosidase with $90 \mathrm{kDa}$. The rhamnosidase in the enzyme mixture from A. niger had $80 \mathrm{kDa}$.

\section{Discussion}

Due to their occurrence as glycosides, the bioavailability and bioactivity of polyphenols are limited, especially for rhamnoglucosides which cannot be cleaved by human intestinal enzymes. A few studies reported the release of rhamnose from flavonoid rhamnoglucoside by gut bacteria [23]. However, information on the rhamnosidase activity of probiotics is still limited. Previous studies have shown the occurrence and the properties of rhamnosidases from 
Table 3 Hydrolysis of rhamnose (bioconversion) from narcissin and rutin in $C$. johnstonii extract at certain time points; percentages of cleaved glycosides. $\mathrm{n}=3$

\begin{tabular}{|c|c|c|c|c|c|c|}
\hline & \multicolumn{6}{|c|}{ Bioconversion (\%) } \\
\hline & \multicolumn{3}{|c|}{ Narcissin } & \multicolumn{3}{|l|}{ Rutin } \\
\hline & 4 days & 7 days & 10 days & 4 days & 7 days & 10 days \\
\hline B. animalis ssp. lactis & $36 \pm 6$ & $72 \pm 1$ & $93 \pm 10$ & $3 \pm 2$ & $28 \pm 1$ & $57 \pm 1$ \\
\hline B. longum ssp. infantis & $47 \pm 1$ & $85 \pm 1$ & $84 \pm 13$ & $15 \pm 1$ & $45 \pm 2$ & $73 \pm 3$ \\
\hline L. acidophilus & $56 \pm 1$ & $77 \pm 4$ & $86 \pm 6$ & $11 \pm 4$ & $36 \pm 4$ & $55 \pm 2$ \\
\hline L. paracasei DN114001 & $22 \pm 2$ & $67 \pm 2$ & $95 \pm 1$ & $3 \pm 1$ & $26 \pm 2$ & $66 \pm 1$ \\
\hline L. paracasei DSM 20312 & $33 \pm 7$ & $54 \pm 15$ & $89 \pm 1$ & $8 \pm 1$ & $21 \pm 3$ & $50 \pm 3$ \\
\hline L. paracasei CRL431 & $22 \pm 1$ & $38 \pm 3$ & $52 \pm 4$ & $2 \pm 2$ & $6 \pm 2$ & $18 \pm 5$ \\
\hline L. reuteri & $14 \pm 9$ & $15 \pm 7$ & $25 \pm 14$ & 0 & 0 & $5 \pm 2$ \\
\hline L. rhamnosus GG & $22 \pm 8$ & $32 \pm 4$ & $72 \pm 3$ & $3 \pm 2$ & $13 \pm 5$ & $38 \pm 11$ \\
\hline L. plantarum & $56 \pm 9$ & $87 \pm 5$ & $94 \pm 3$ & $6 \pm 0$ & $16 \pm 1$ & $26 \pm 2$ \\
\hline L. brevis & $40 \pm 5$ & $79 \pm 7$ & $88 \pm 8$ & $10 \pm 2$ & $38 \pm 11$ & $64 \pm 11$ \\
\hline L. delbrueckii ssp. bulgaricus & $38 \pm 4$ & $47 \pm 3$ & $81 \pm 15$ & $4 \pm 1$ & $20 \pm 6$ & $32 \pm 6$ \\
\hline Lc. lactis ssp. lactis & $31 \pm 8$ & $69 \pm 7$ & $98 \pm 5$ & $6 \pm 1$ & $45 \pm 13$ & $62 \pm 14$ \\
\hline L. fermentum & $42 \pm 3$ & $87 \pm 7$ & $97 \pm 1$ & $12 \pm 0$ & $51 \pm 13$ & $78 \pm 9$ \\
\hline S. salivarius ssp. thermophilus & $42 \pm 7$ & $60 \pm 9$ & $71 \pm 10$ & $7 \pm 0$ & $21 \pm 4$ & $33 \pm 0$ \\
\hline
\end{tabular}

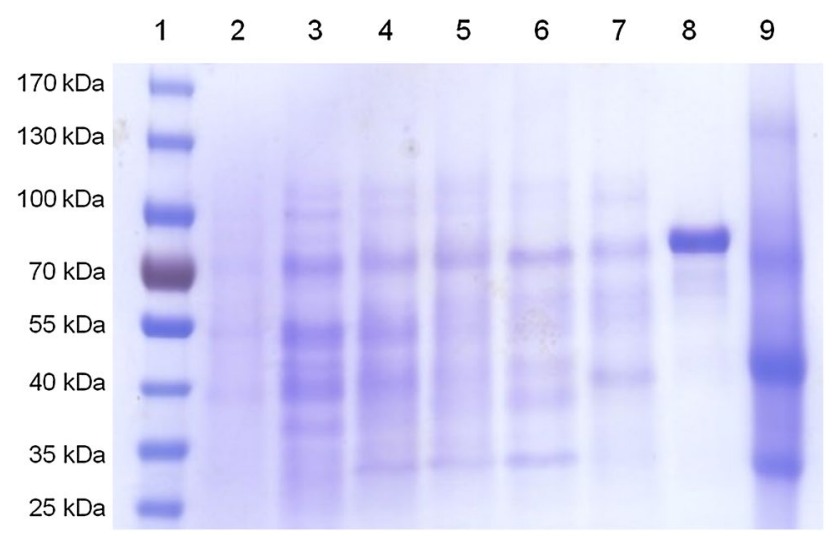

Fig. 2 SDS-PAGE: (1) marker; (2) lysate of L. acidophilus without induction by NPRP; lysate of $L$. acidophilus with induction using NPRP after incubation for (3) 10 days, (4) 7 days, (5) 4 days, (6) one day, (7) without incubation; (8) recombinant rhamnosidase (20 mg/ $\mathrm{mL}$ ); (9) enzyme mixture from A. niger (cellulase; $75 \mathrm{mg} / \mathrm{mL}$ )

L. plantarum, L. acidophilus [24, 25] and Pediococcus acidilactici [26]. Another study has shown the hydrolysis of hesperidin by 6 out of 33 bifidobacteria strains within $48 \mathrm{~h}$ [6]. The results from our study provide further evidence of rhamnosidase activity in probiotics and of its substrate specificity. Rhamnosidase activity was found in all strains as shown with NPRP as substrate although with some differences of the activity among the strains. However, naringin could not be cleaved by any strain; rutin was cleaved by four strains to an extent below 5\%, whereas the hydrolysis of narcissin and hesperidin was more rapid and to a higher extent. The preferred cleavage of hesperidin in contrast to rutin is consistent with the results from Amaretti et al. [6].
The preference for cleaving hesperidin (1-6 bonds) and no activity on naringin (1-2 bonds) conforms to previous studies with Pediococcus acidilactici or L. plantarum [25, 26]. In contrast to these and our results, Bang et al. [27] isolated a bifidobacterium (B. dentium) from human faeces samples with the ability to hydrolyse also rhamnose from the neohesperidose in naringin. Interestingly, this strain also was more effective in hydrolysing (1-6) bonds than (1-2) linkages of rhamnoglycosides.

In case of hesperidin no glucoside was detected, only the aglycone was found so the cleavage may be at least partly caused by rutinosidase. So far only fungal rutinosidase is known, so the contribution of bacterial rutinosidase has to be confirmed in further studies.

When comparing the results of Amaretti et al. [6] and our study, the conclusion can be drawn that lactobacilli are more effective in the cleavage of rhamnose from rutin and hesperidin than bifidobacteria, at least those used by Amaretti et al. [6]. A high rhamnosidase activity correlates well with the ability of cleaving hesperidin as shown for L. brevis, Lc lactis ssp. lactis and L. fermentum. A low rhamnosidase activity mostly correlates with a low hydrolysis rate of hesperidin as seen for L. reuteri. Interestingly, L. rhamnosus GG has a high rhamnosidase activity but low hydrolysis rate of hesperidin, indicating that the substrate specificity of the rhamnosidase enzyme from different probiotics differs. In strains with a high rhamnosidase activity as determined using NPRP, the probability for an induction of the rhamnose expression by narcissin and a following hydrolysis of rutin is increased. This was shown for $L c$. lactis, $L$. fermentum or B. longum ssp. infantis. For L. reuteri, a low rhamnosidase activity (as shown with NPRP) correlates with 
a very low induction of the rhamnosidase using narcissin and a low following cleavage of rutin. Again, for L. rhamnosus GG no high induction of the rhamnosidase activity using narcissin was found even if the rhamnosidase activity using NPRP was high.

Here, the recombinant $\alpha$-L-rhamnosidase was active towards hesperidin consistent to [28] and rutin, both of them show $\alpha-1,6$ linkages to the $\beta$-D-glucosides. However, the enzyme was not active towards naringin, in which the L-rhamnose is $\alpha-1,2$ linked to the $\beta$-D-glucoside. Interestingly, naringin is cleaved by rhamnosidase from A. niger. This goes conform with previous studies showing rhamnosidases from fungi to hydrolyse naringin [29]. The molecular weight of the rhamnosidase from A. niger and the probiotics or the recombinant one are different, potentially indicating a distinct glycosylation pattern which may lead to different substrate specificities.

Hydrolysis of the rhamnose from the main compounds (rutin, narcissin) of a $C$. johnstonii extract may increase bioactivity and bioavailability. The hydrolysis of narcissin by probiotics is efficiently and interestingly induces the activity of probiotic rhamnosidase so that rutin is also cleaved by all strains. Narcissin may be used as inducers for other flavonoid rhamnoglucosides as well. An induction of rhamnosidase activity by glycosides containing L-rhamnosyl moieties was suggested previously and shown for rutin by gut bacteria $[25,30]$. In contrast to these previous studies, one easily hydrolysed compound of a plant extract helps to induce the cleavage of another compound in this extract. In such a way, one compound in the extract increases the bioavailability of another compound in this extract having a synergistic effect.

Considering the transit time of food in the gastro-intestinal tract, the velocity of hydrolysis by gut bacteria in the proximal as well as distal colon is relevant for hesperidin and narcissin. A pre-incubation of a flavonol glycoside containing supplement with bacteria may increase the yield of hydrolysis and thus bioavailability tremendously.

\section{Conclusion}

14 probiotics hydrolysed hesperidin and narcissin, four probiotics hydrolysed a small amount of rutin, but no strain was able to cleave naringin. The efficiency of hydrolysis and substrate specificity was strain dependent. Recombinant $\alpha$-Lrhamnosidase cleaved all rhamnoglucosides except naringin which was only cleaved by rhamnosidase from $A$. niger. One of the main compounds from $C$. johnstonii extract, narcissin, was not only hydrolysed by probiotic rhamnosidase, but induced rhamnosidase activity for cleavage of rutin. These findings provide further evidence of rhamnosidase activity in probiotics, making them to attractive sources for combined products with plant extracts with increased bioavailability of the active compounds.

Acknowledgements Open access funding provided by University of Vienna. Thanks to CMU for providing $C$. johnstonii powder. Parts of this work were supported by short term grant from the ASEANEuropean Academic University Network (ASEA-UNINET) and by a grant of the City of Vienna HJST (Hochschuljubiläumsstiftung).

\section{Compliance with ethical standards}

Conflict of interest The authors declare that they have no conflicts of interest.

Open Access This article is distributed under the terms of the Creative Commons Attribution 4.0 International License (http://creativecommons.org/licenses/by/4.0/), which permits unrestricted use, distribution, and reproduction in any medium, provided you give appropriate credit to the original author(s) and the source, provide a link to the Creative Commons license, and indicate if changes were made.

\section{References}

1. Middleton E Jr (1998) Effect of plant flavonoids an immune and inflammatory cell function. In: Flaonoids in the living system; Springer, Berlin, pp 175-182

2. DuPont MS, Day AJ, Bennett RN, Mellon FA, Kroon PA (2004) Absorption of kaempferol from endive, a source of kaempferol3-glucuronide, in humans. Eur J Clin Nutr 58:947-954

3. Harborne JB, Williams CA (2000) Advances in flavonoid research since 1992. Phytochem 55:481-504

4. Manach C, Scalbert A, Morand C, Rémésy C, Jiménez L (2004) Polyphenols: food sources and bioavailability. Am J Clin Nutr 79:727-747

5. Hertog MGL, Hollman PCH, Venema DP (1992) Optimization of a quantitative HPLC determination of potentially anticarcinogenic flavonoids in vegetables and fruits. J Agric Food Chem 40:1591-1598

6. Amaretti A, Raimondi S, Leonardi A, Quartieri A, Rossi M (2015) Hydrolysis of the rutinose-conjugates flavonoids rutin and hesperidin by the gut microbiota and bifidobacteria. Nutrients 7:2788-2800

7. Erlund I (2004) Review of the flavonoids quercetin, hesperetin, and naringenin. Dietary sources, bioactivities, bioavailability, and epidemiology. Nutr Res 24:851-874

8. Manach C, Williamson G, Morand C, Scalbert A, Rémésy C (2005) Bioavailability and bioefficacy of polyphenols in humans. I. Review of 97 bioavailability studies. Am J Clin Nutr 81:230S-242S

9. Mueller M, Hobiger S, Jungbauer A (2010) Anti-inflammatory activity of extracts from fruits, herbs and spices. Food Chem 122:987-996

10. Russo M, Spagnuolo C, Tedesco I, Bilotto S, Russo GL (2012) The flavonoid quercetin in disease prevention and therapy: facts and fancies. Biochem Pharmacol 83:6-15

11. Parhiz H, Roohbakhsh A, Soltani F, Rezaee R, Iranshahi M (2015) Antioxidant and anti-inflammatory properties of the citrus flavonoids hesperidin and hesperetin: an updated review of their molecular mechanisms and experimental models. Phytother Res 29:323-331

12. Patel K, Singh GK, Patel DK (2012) A review on pharmacological and analytical aspects of naringenin. Chin J Integr Med 1-12 
13. Nielsen ILF, Chee WSS, Poulsen L, Offord-Cavin E, Rasmussen SE, Frederiksen H, Enslen M, Barron D, Horcajada MN, Williamson $\mathrm{G}$ (2006) Bioavailability is improved by enzymatic modification of the citrus flavonoid hesperidin in humans: a randomized, double-blind, crossover trial. J Nutr 136:404-408

14. Amaro IM, Rocha J, Vila-Real H, Eduardo-Figueira M, MotaFilipe H, Sepodes B, Ribeiro MH (2009) Anti-inflammatory activity of naringin and the biosynthesised naringenin by naringinase immobilized in microstructured materials in a model of DSSinduced colitis in mice. Food Res Int 42:1010-1017

15. de Araújo ME, Moreira Franco YE, Alberto TG, Sobreiro MA, Conrado MA, Priolli DG, Frankland Sawaya AC, Ruiz AL, de Carvalho JE, de Oliveira Carvalho P (2013) Enzymatic de-glycosylation of rutin improves its antioxidant and antiproliferative activities. Food Chem 141:266-273

16. Kaur A, Singh S, Singh RS, Schwarz WH, Puri M (2010) Hydrolysis of citrus peel naringin by recombinant $\alpha$-L-rhamnosidase from Clostridium stercorarium. J Chem Technol Biotechnol 85:1419-1422

17. Soria F, Ellenrieder G (2002) Thermal inactivation and product inhibition of Aspergillus terreus CECT $2663 \alpha$-L-rhamnosidase and their role on hydrolysis of naringin solutions. Biosci Biotechnol Biochem 66:1442-1449

18. Spagna G, Barbagallo RN, Martino A, Pifferi PG (2000) A simple method for purifying glycosidases: $\alpha$-L-rhamnopyranosidase from Aspergillus niger to increase the aroma of Moscato wine. Enz Microbial Technol 27:522-530

19. Yadav V, Yadav PK, Yadav S, Yadav KDS (2010) $\alpha-\mathrm{L}-$ Rhamnosidase: a review. Process Biochem 45:1226-1235

20. Gupta C, Prakash D (2014) Phytonutrients as therapeutic agents. J Complement Integr Med 11:151-169

21. Okonogi S, Khonkarn R, Mankhetkorn S, Unger FM, Viernstein H (2013) Antioxidant activity and cytotoxicity of Cyrtosperma johnstonii extracts on drug sensitive and resistant leukemia and small cell lung carcinoma cells. Pharm Biol 51:329-338
22. Mueller M, Reiner J, Fleischhacker L, Viernstein H, Loeppert R, Praznik W (2016) Prebiotic effect of fructans depending on polymerization degree and structure. J Funct Foods 24:264-275

23. Yu K, Jang I, Kang K, Sung C, Kim D (1997) Metabolism of saikosaponin $\mathrm{c}$ and naringin by human intestinal bacteria. Arch Pharm Res 20:420-424

24. Beekwilder J, Marcozzi D, Vecchi S, de Vos R, Janssen P, Francke C, van Hylckama Vlieg J, Hall R (2009) Characterization of Rhamnosidases from Lactobacillus plantarum and Lactobacillus acidophilus. Appl Environ Microbiol 75:3447-3454

25. Avila M, Jaquet M, Moine D, Requena T, Peláez C, Arigoni F, Jankovic I (2009) Physiological and biochemical characterization of the two alpha-L-rhamnosidases of Lactobacillus plantarum NCC245. Microbiol 155:2739-2749

26. Michlmayr H, Brandes W, Eder R, Schümann C, del Hierro A, Kulbe K (2011) Characterization of two distinct glycosyl hydrolase family 78 alpha-L-rhamnosidases from Pediococcus acidilactici. Appl Environ Microbiol 77:6524-6530

27. Bang S, Hyun Y, Shim J, Hong S, Kim D (2015) Metabolism of rutin and poncirin by human intestinal microbiota and cloning of their metabolizing $\alpha$-L-rhamnosidase from Bifidobacterium dentium. J Microbiol Biotechnol 25:18-25

28. Zhang R, Zhang BL, Xie T, Li GC, Tuo Y, Xiang YT (2015) Biotransformation of rutin to isoquercitrin using recombinant $\alpha$-L-rhamnosidase from Bifidobacterium breve. Biotechnol Lett 37:1257-1264

29. Liu Q, Lu L, Xiao M (2012) Cell surface engineering of $\alpha$-Lrhamnosidase for naringin hydrolysis. Bioresour Technol 123:144-149

30. Shin NR, Moon JS, Shin SY, Li L, Lee YB, Kim TJ, Han NS (2015) Isolation and characterization of human intestinal Enterococcus avium EFEL009 converting rutin to quercetin. Lett Appl Microbiol 62:68-74 